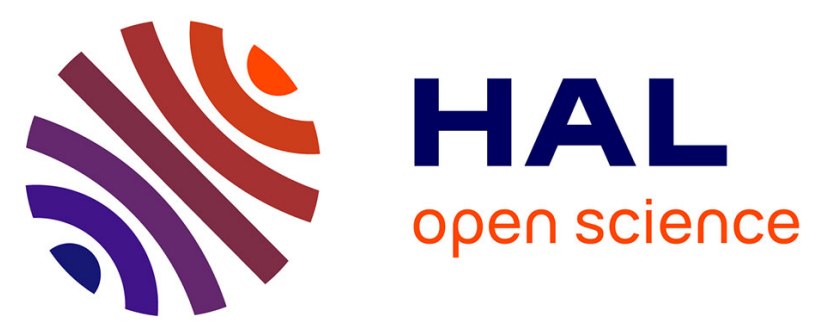

\title{
Benchmark 3D: a version of the DDFV scheme with cell/vertex unknowns on general meshes
}

Boris Andreianov, Florence Hubert, Stella Krell

\section{To cite this version:}

Boris Andreianov, Florence Hubert, Stella Krell. Benchmark 3D: a version of the DDFV scheme with cell/vertex unknowns on general meshes. Finite Volumes for Complex Applications VI, Jun 2011, Prague, Czech Republic. pp.937-948, 10.1007/978-3-642-20671-9_91 . hal-00572732

\section{HAL Id: hal-00572732 \\ https://hal.science/hal-00572732}

Submitted on 2 Mar 2011

HAL is a multi-disciplinary open access archive for the deposit and dissemination of scientific research documents, whether they are published or not. The documents may come from teaching and research institutions in France or abroad, or from public or private research centers.
L'archive ouverte pluridisciplinaire HAL, est destinée au dépôt et à la diffusion de documents scientifiques de niveau recherche, publiés ou non, émanant des établissements d'enseignement et de recherche français ou étrangers, des laboratoires publics ou privés.

\section{다)(1) $(5$}

Distributed under a Creative Commons Attribution - NonCommerciall 4.0 International 


\title{
Benchmark 3D: a version of the DDFV scheme with cell/vertex unknowns on general meshes.
}

\author{
Boris Andreianov, Florence Hubert and Stella Krell
}

\begin{abstract}
This paper gives numerical results for a 3D extension of the 2D DDFV scheme. Our scheme is of the same inspiration as the one called CeVe-DDFV ([9]), with a more straightforward dual mesh construction. We sketch the construction in which, starting from a given 3D mesh (which can be non conformal and have arbitrary polygonal faces), one defines a dual mesh and a diamond mesh, reconstructs a discrete gradient, and proves the discrete duality property. Details are given in [1].
\end{abstract}

\section{DDFV methods in 2D and in 3D. A 3D CeVe-DDFV scheme.}

DDFV ("Discrete Duality Finite Volume") scheme was introduced in 2D by Hermeline in [15] and by Domelevo and Omnès in [13]. To handle anisotropic problems or nonlinear problems, or in order to work on general distorted meshes, full gradient reconstruction from point values is a popular strategy. It is well known that reconstruction of a discrete gradient is facilitated by adding unknowns that are new with respect to those of standard cell-centered finite volume schemes. The 2D DDFV method consists in adding new unknowns at the vertices of the initial mesh (this initial mesh is often called the primal one), and in use of new control volumes (called dual cells, or co-volumes) around these points. A family of diamond cells is naturally associated to this construction, each diamond being built on two neighbor cell centers $x_{K}, x_{L}$ and the two vertices of the edge $K \mid L$ that separates them. On a diamond, one can construct a discrete gradient direction per direction (cell-cell and vertex-vertex), following the idea of [8]. It turns out that this discrete gradient is related by a discrete analogue of integraton-by-parts formula, called "discrete du-

Boris Andreianov

CNRS UMR 6623, Besancon, France, e-mail: boris. andreianov@univ-fcomte.fr

Florence Hubert

LATP, Université de Provence, Marseille, France, e-mail: fhubert@cmi . univ-mrs . fr

Stella Krell

INRIA, Lille, France, e-mail: stella.krelleinria.fr 
ality", to the classical discrete finite volume divergence associated with these two families of meshes. This duality property greatly simplifies the theoretical analysis of finite volume schemes based on the DDFV construction, see e.g. [5, 2]. This 2D strategy reveals to be particularly efficient in terms of gradient approximation (see $[7,14]$ ) and has been extended to a wide class of PDE problems (see [1, 5, 6, 18, 19] and references therein).

The 3D CeVe-DDFV scheme we present here also keeps unknowns only at the cell centers and the vertices of the primal mesh, and it uses the primal mesh, a dual mesh and a diamond mesh; as in the 2D case, a diamond is constructed from two neighbor cell centers $x_{K}, x_{L}$ and from $l$ vertices of the edge $K \mid L$ that separates them $(l \geq 3)$. The price to pay is that the gradient reconstruction becomes more intricate. As in 2D, one direction per diamond is reconstructed using the two cell center unknowns at the nodes $x_{K}, x_{L}$; two complementary directions of the gradient in $K \mid L$ are reconstructed simultaneously, by a suitable interpolation of the vertex values in each face $K \mid L$ of the primal mesh. While the case $l=3$ (meshes with triangular faces) offers no choice, in general we have to fix a formula for interpolation that is consistent with affine functions and which leads to discrete duality (with respect to appropriately defined dual cells). This was achieved independently in [17] and in $[3,4,1]$, with two different approaches (the above description stems from the point of view developed in $[3,4,1])$.

Several 3D DDFV constructions exist. The CeVe-DDFV scheme by Pierre et al. (see [12]) was the pioneering work in 3D; a particular feature of this method was in the double covering of the domain by the dual mesh. This approach led to a method that is only slightly different from ours; we refer to the benchmark paper [9] in the same collection. Next, Hermeline in [16] introduced the important idea to associate additional unknowns with the face centers of the primal mesh. In the subsequent work [17] of Hermeline, elimination of these unknowns eventually led to the same method that the one we describe. Many numerical tests are given in [16, 17]. Finally, Coudière and Hubert in [10] introduced edge unknowns, instead of eliminating face unknowns. This idea assessed a new strategy of 3D DDFV approximation; we call it CeVeFE-DDFV because with respect to the primal mesh, cell, vertex and face+edge unknowns are used. Let us point out the differences with respect to CeVe-DDFV strategies. In [10], each diamond is constructed on two cell centers $x_{K}, x_{L}$, on two vertices $x_{K^{*}}, x_{L^{*}}$ in the face $K \mid L$, and one face center $x_{K L} \in K \mid L$ and one edge center $x_{K^{*} L^{*}} \in\left[x_{K^{*}}, x_{L^{*}}\right]$. Then the gradient is reconstructed per direction (cell-cell, vertexvertex and face-edge), as in $2 \mathrm{D}$. The edge and face centers are the centers for a new, third mesh. The CeVeFE-DDFV method is the object of the benchmark paper [11] in the same collection.

Let us present the construction of our 3D CeVe-DDFV scheme. The primal mesh needs not be conformal; there is no restriction on number of faces or face edges. For simplicity, let us assume that the primal mesh volumes are convex; that their centers belong to the volumes; and the face centers belong to the faces. These restrictions can be relaxed, see [1]; but let us stress that the edge points must be the middlepoints. 
Notation. We use a triple $\mathfrak{T}=\left(\overline{\mathfrak{M}^{o}}, \overline{\mathfrak{M}^{*}}, \mathfrak{D}\right)$ of partitions of $\Omega$ into polyhedra.

- $\mathfrak{M}^{o}$ denotes the initial mesh ${ }^{1}$, called primal mesh. We call $\partial \mathfrak{M}^{o}$ the set of all faces of this mesh that are included in $\partial \Omega$. These faces are considered as flat boundary (primal) control volumes. We denote by $\overline{\mathfrak{M}^{o}}$ the union $\mathfrak{M}^{o} \cup \partial \mathfrak{M}^{o}$.

- Center: To any (primal) control volume $K \in \overline{\mathfrak{M}^{o}}$, we associate a point $x_{K} \in K$.

- Vertex: A generic vertex of $\overline{\mathfrak{M}^{o}}$ is denoted by $x_{K^{*}}$.

- Neighbors: given $K \in \mathfrak{M}^{o}$, all control volumes $L \in \overline{\mathfrak{M}^{o}}$ such that $K$ and $L$ have a common face (or part of a face) form the set $\mathscr{N}(K)$ of neighbors of $K$.

- Face: for all $L \in \mathscr{N}(K)$, by $K \mid L$ we denote $\partial K \cap \partial L$ which is a face (or a part of a face) of the mesh $\mathfrak{M}^{o}$; it is supplied with a face center $x_{K L} \in K \mid L$.

- Edge: An egde $\left[x_{K^{*}}, x_{L^{*}}\right]$ of $\overline{\mathfrak{M}^{o}}$ is defined by two neighbor vertices $x_{K^{*}}, x_{L^{*}}$; it is marked with the center $x_{K^{*} L^{*}}$ that must be its middlepoint $\left(x_{K^{*}}+x_{L^{*}}\right) / 2$.

- Element: An element $T=T_{K^{*} ; L^{*}}^{K ; L}$ is the tetrahedron $\left(x_{K}, x_{K \mid L}, x_{K^{*} \mid L^{*}}, x_{K^{*}}\right)$ : here $K$ is a primal volume ; $K \mid L$ is a face of $K$; and $\left[x_{K^{*}}, x_{L^{*}}\right]$ is an edge of $K \mid L$ (see Fig. 1). The set of all elements is denoted by $\mathscr{T}$. If $x_{K}$ is a vertice of $T \in \mathscr{T}$, then we say that $T$ is associated ${ }^{2}$ with the volume $K$, and we write $T \sim K$.

- $\overline{\mathfrak{M}^{*}}$ denotes the dual mesh constructed as follows. A generic vertex $x_{K^{*}}$ of $\mathfrak{M}^{o}$ is associated with the polyhedron $K^{*} \in \overline{\mathfrak{M}^{*}}$ made of all elements $T \in \mathscr{T}$ that share the vertex $x_{K^{*}}$ (we write $T \sim K^{*}$ ). If $x_{K^{*}} \in \Omega$, we say that $K^{*}$ is a dual control volume and write $K^{*} \in \mathfrak{M}^{*}$; and if $x_{K^{*}} \in \partial \Omega$, we say that $K^{*}$ is a boundary dual control volume and write $\kappa^{*} \in \partial \mathfrak{M}^{*}$. Thus $\overline{\mathfrak{M}^{*}}=\mathfrak{M}^{*} \cup \partial \mathfrak{M}^{*}$.

- $\mathfrak{D}$ is the diamond mesh. For $K \in \mathfrak{M}^{0}, L \in \mathscr{N}(K)$, the union of the convex hull of $x_{K}$ and $K \mid L$ with the convex hull of $x_{L}$ and $K \mid L$ is called diamond, denoted by $D^{K L}$.

For expression of the discrete operators one needs a convention on diamond orientation, subdiamonds and other objects and notation of [1]; we give them via Fig. 1.
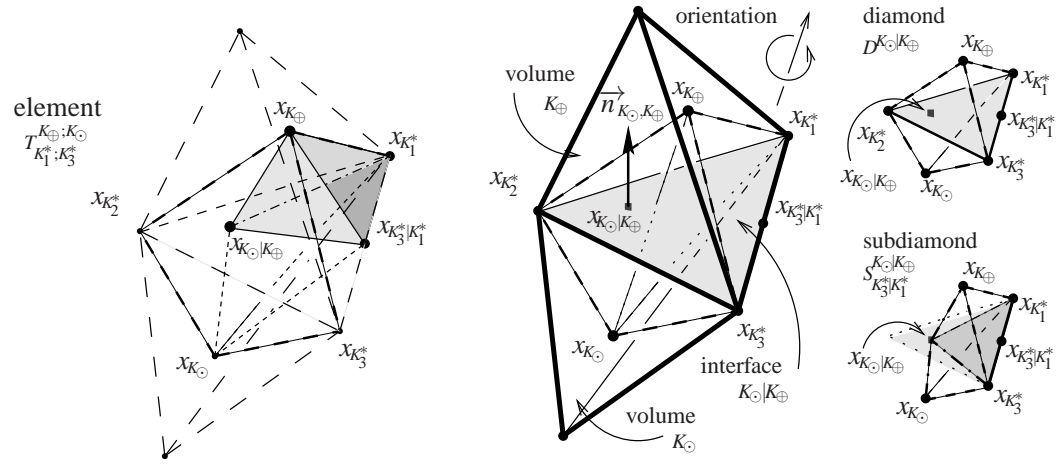

Fig. 1 Element (left). Oriented diamond, subdiamond and related notation, cf. [1] (right).

\footnotetext{
${ }^{1}$ This means, $\mathfrak{M}^{o}$ is one of the meshes provided by the benchmark organizers.

${ }^{2}$ Because we have made the assumption that $x_{K L} \in K \mid L$, the relation $T \sim K$ simply means that $T$ is included in $K$. The same observation applies to the notation $T \sim K^{*}$. See [1] for generalizations.
} 


\section{Discrete space and discrete operators; the discrete duality feature.}

- A discrete function on $\Omega$ is a set $w^{\mathfrak{T}}=\left(w^{\mathfrak{M}^{o}}, w^{\mathfrak{M}^{*}}\right)$ consisting of two sets of real values $w^{\mathfrak{M}^{o}}=\left(w_{K}\right)_{K \in \mathfrak{M}^{o}}$ and $w^{\mathfrak{M}^{*}}=\left(w_{K^{*}}\right)_{K^{*} \in \mathfrak{M}^{*}}$.

- A discrete function on $\bar{\Omega}$ is a set $w^{\overline{\mathfrak{T}}}=\left(w^{\mathfrak{M}^{o}}, w^{\mathfrak{M}^{*}} ; w^{\partial \mathfrak{M}^{o}}, w^{\partial \mathfrak{M}^{*}}\right) \equiv\left(w^{\mathfrak{T}} ; w^{\partial \mathfrak{T}}\right)$, $w^{\mathfrak{M}^{o}}=\left(w_{K}\right)_{K \in \mathfrak{M}^{o}}, w^{\mathfrak{M}^{*}}=\left(w_{K^{*}}\right)_{K^{*} \in \mathfrak{M}^{*}}, w^{\partial \mathfrak{M}^{o}}=\left(w_{K}\right)_{K \in \partial \mathfrak{M}^{o}}, w^{\partial \mathfrak{M}^{*}}=\left(w_{K^{*}}\right)_{K^{*} \in \partial \mathfrak{M}^{*}}$.

- A discrete field on $\Omega$ is a set $\overrightarrow{\mathscr{F}}^{\mathfrak{T}}=\left(\overrightarrow{\mathscr{F}}_{D}\right)_{D \in \mathfrak{D}}$ of vectors of $\mathbb{R}^{3}$.

- We write $\mathbb{R}^{\mathfrak{T}}, \mathbb{R}^{\overline{\mathfrak{T}}},\left(\mathbb{R}^{3}\right)^{\mathfrak{D}}$, respectively, for the sets of discrete functions/fields.

- Discrete divergence is the operator acting from $\left(\mathbb{R}^{3}\right)^{\mathfrak{D}}$ to $\mathbb{R}^{\mathfrak{T}}$, given by

$$
\operatorname{div}^{\mathfrak{T}}: \overrightarrow{\mathscr{F}}^{\mathfrak{T}} \mapsto\left(\left(\operatorname{div}_{K} \overrightarrow{\mathscr{F}^{\mathfrak{T}}}\right)_{K \in \mathfrak{M}^{o}},\left(\operatorname{div}_{K^{*}} \overrightarrow{\mathscr{F}}^{\mathfrak{T}}\right)_{K^{*} \in \mathfrak{M}^{*}}\right)=: \operatorname{div}^{\mathfrak{T}} \overrightarrow{\mathscr{F}}^{\mathfrak{T}},
$$

where the entries $\operatorname{div}_{K} \overrightarrow{\mathscr{F}}^{\mathfrak{T}}, \operatorname{div}_{K^{*}} \overrightarrow{\mathscr{F}}^{\mathfrak{T}}$ of the discrete function $\operatorname{div}^{\mathfrak{T}} \overrightarrow{\mathscr{F}}^{\mathfrak{T}}$ on $\Omega$ are

$$
\operatorname{div}_{K} \overrightarrow{\mathscr{F}}^{\mathfrak{T}}=\frac{1}{\operatorname{Vol}(K)} \sum_{T \sim K} m_{T} \overrightarrow{\mathscr{F}}_{T} \cdot \vec{n}_{T}, \quad \operatorname{div}_{K^{*}} \overrightarrow{\mathscr{F}}^{\mathfrak{T}}=\frac{1}{\operatorname{Vol}\left(K^{*}\right)} \sum_{T \sim K^{*}} m_{T}^{*} \overrightarrow{\mathscr{F}}_{T} \cdot \vec{n}_{T}^{*}
$$

$\vec{n}_{T}, \vec{n}_{T}^{*}$ being the exterior normal vectors to $\partial_{K}, \partial_{K^{*}}$. Formulae (2) stem from the standard procedure of finite volume discretization, applied on $\mathfrak{M}^{\circ}$ and on $\mathfrak{M}^{*}$.

- Discrete gradient is the operator acting from $\mathbb{R}^{\overline{\mathfrak{T}}}$ to $\left(\mathbb{R}^{3}\right)^{\mathfrak{D}}$, given by

$$
\vec{\nabla}^{\mathfrak{T}}: w^{\overline{\mathfrak{T}}} \mapsto\left(\vec{\nabla}_{D} w^{\overline{\mathfrak{T}}}\right)_{D \in \mathfrak{D}}=: \vec{\nabla}^{\mathfrak{T}} w^{\overline{\mathfrak{T}}}
$$

where the entry $\vec{\nabla}_{D} w^{\overline{\mathfrak{T}}}$ of the discrete field $\vec{\nabla}^{\mathfrak{T}} w^{\overline{\mathfrak{T}}}$ corresponding to $D=D^{K_{\odot} \mid K_{\oplus}}$ (see Fig. 1) is reconstructed from the values $w_{K_{\odot}}, w_{K_{\oplus}}$ at the neighbor centers $x_{K_{\odot}}, x_{K_{\oplus}}$ (they give the projection on $\overrightarrow{x_{K_{\odot}} x_{K_{\oplus}}}$ ) and the values $\left(w_{K_{i}^{*}}\right)_{i=1}^{l}$ at the $l$ vertices of the interface $K_{\odot} \mid K_{\oplus}$ (they give the projection on the plane $\left.K_{\odot} \mid K_{\oplus}\right)^{3}$ with

$$
\begin{aligned}
& \vec{\nabla}_{D} w^{\overline{\mathfrak{T}}}=\frac{1}{6 \operatorname{Vol}(D)} \sum_{i=1}^{l}\left\{\frac{\left\langle\overrightarrow{x_{K_{\odot}} x_{K_{\oplus}}}, \overrightarrow{\left.x_{K_{\odot} \mid K_{\oplus}} x_{K_{i}^{*}}\right|_{K_{i+1}^{*}}}, \overrightarrow{x_{K_{i}^{*}} x_{K_{i+1}^{*}}}\right\rangle}{\overline{x_{K_{\odot}} x_{K_{\oplus}}} \cdot \vec{n}_{K_{\odot}, K_{\oplus}}}\left(w_{K_{\oplus}}-w_{K_{\odot}}\right) \vec{n}_{K_{\odot}, K_{\oplus}}\right. \\
& \left.+2\left(w_{K_{i+1}^{*}}-w_{K_{i}^{*}}\right)\left[\overrightarrow{x_{K_{\odot}} x_{K_{\oplus}}} \times \overrightarrow{x_{K_{\odot} \mid K_{\oplus}} x_{K_{i}^{*} \mid K_{i+1}^{*}}}\right]\right\} .
\end{aligned}
$$

- Pick $\llbracket w^{\mathfrak{T}}, v^{\mathfrak{T}} \rrbracket:=\frac{1}{3} \sum_{K \in \mathfrak{M}^{o}} \operatorname{Vol}(K) w_{K} v_{K}+\frac{2}{3} \sum_{K^{*} \in \mathfrak{M}^{*}} \operatorname{Vol}\left(K^{*}\right) w_{K^{*}} v_{K^{*}}$ for scalar product on $\mathbb{R}^{\mathfrak{T}}$ and $\left\{\left\{\overrightarrow{\mathscr{F}^{\mathfrak{T}}}, \overrightarrow{\mathscr{G}^{\mathfrak{T}}}\right\}\right\}:=\sum_{D \in \mathfrak{D}} \operatorname{Vol}(D) \overrightarrow{\mathscr{F}_{D}} \cdot \overrightarrow{\mathscr{G}_{D}}$ for scalar product on $\left(\mathbb{R}^{3}\right)^{\mathfrak{D}}$. And now, one can mimic the identity $-\int_{\Omega}(\operatorname{div} \overrightarrow{\mathscr{F}}) w=\int_{\Omega} \overrightarrow{\mathscr{F}} \cdot \vec{\nabla} w$ for $\left.w\right|_{\partial \Omega}=0$ :

Proposition 1 (the discrete duality property; see [3, 1], see also [17]). For all $\overrightarrow{\mathscr{F}^{\mathfrak{T}}} \in\left(\mathbb{R}^{3}\right)^{\mathfrak{D}}$ and all $w^{\overline{\mathfrak{T}}} \in \mathbb{R}^{\overline{\mathfrak{T}}}$ with $w^{\partial \mathfrak{T}}=0, \quad \llbracket-\operatorname{div}^{\mathfrak{T}^{\mathfrak{T}}} \overrightarrow{\mathscr{F}^{\mathfrak{T}}}, w^{\mathfrak{T}} \rrbracket=\left\{\left\{\overrightarrow{\mathscr{F}^{\mathfrak{T}}}, \vec{\nabla}{ }^{\mathfrak{T}} w^{\overline{\mathfrak{T}}}\right\}\right.$.

\footnotetext{
${ }^{3}$ When $l=3$, one simply uses the three-point interpolation in the plane $K_{\odot} \mid K_{\oplus}$ to reconstruct this projection. Clearly, the interpolation is exact for affine functions. In general, the reconstruction (3), which is exact for affine functions, is based upon the 2D identity given in [3] and [1, Appendix].
} 
The scheme. In this benchmark, one approximates the linear diffusion problem $-\operatorname{div}[\mathbf{A}(\cdot) \vec{\nabla} u]=f(\cdot)$ with Dirichlet boundary condition $\left.u\right|_{\partial \Omega}=\bar{u}(\cdot), \mathbf{A}(\cdot)$ being a heterogeneous anisotropic diffusion tensor and $f(\cdot)$ being a source term. Let $\mathbb{P}^{\mathfrak{T}}$ denote the projection on the DDFV mesh $\mathfrak{T}$ (i.e. the components of $\mathbb{P}^{\mathfrak{T}} f$ are the mean values of $f \in L^{1}(\Omega)$ per primal and per dual volumes); $\mathbb{P}^{\partial \mathfrak{T}}$ is the projection on the boundary part of the mesh. Let $\overrightarrow{\mathbb{P}}^{\mathfrak{T}}$ denote the projection on the diamond mesh $\mathfrak{D}$. For general data, the heterogeneity of the matrix $\mathbf{A}(\cdot)$ is taken into account by using the diamond-wise projection $\mathbf{A}^{\mathfrak{T}}:=\overrightarrow{\mathbb{P}}^{\mathfrak{T}} \mathbf{A}(\cdot)$; similarly, we use $f^{\mathfrak{I}}=\mathbb{P}^{\mathfrak{T}} f(\cdot)$ as the discrete source term. The boundary condition is given by the projection $\mathbb{P}^{\partial \mathfrak{T}} \bar{u}(\cdot)$.

For a fully practical discretization of $\mathbf{A}(\cdot)$ and $f(\cdot)$ (which are continuous in all the tests we perform), for every element (recall that diamonds, primal volumes and dual volumes of a DDFV mesh are unions of elements, see Fig. 1) we take the mean value of the four vertices of the element. The point values of the exact solution $u_{e}$ in the centers of the boundary volumes are used as discrete boundary conditions.

Given a DDFV mesh $\mathfrak{T}$ of $\Omega$ the method writes as:

$$
\text { Find } u^{\mathfrak{T}} \text { s.t. }-\operatorname{div}^{\mathfrak{T}}\left[\mathbf{A}^{\mathfrak{T}} \vec{\nabla}^{\mathfrak{T}} u^{\overline{\mathfrak{T}}}\right]=f^{\mathfrak{T}} \text { with } u^{\overline{\mathfrak{T}}}=\left(u^{\mathfrak{T}} ; \mathbb{P}^{\partial \mathfrak{T}} \bar{u}\right) \text {. }
$$

Convergence. From the discrete duality (Prop. 1) which is a cornerstone of DDFV schemes, and from consistency properties of the projection, gradient and divergence operators (see [2]; cf. [5] for analogous properties in 2D) one easily derives that the scheme is well posed for $l \leq 4 .{ }^{4}$ Given a family $\left(\mathfrak{T}_{h}\right)_{h}$ of CeVe-DDFV meshes, the associated discrete solutions $u^{\overline{\mathfrak{s}}_{h}}$ enjoy a uniform discrete $H^{1}$ estimate, and they converge to the exact solution $u$ as the size $h$ of the mesh tends to zero. Convergence analysis requires mild proportionality assumptions on the meshes $\boldsymbol{T}_{h}$ in use, see [2].

\section{Numerical results}

In this section, we describe the results obtained on Tests $1-4$ of the benchmark. Notice that, while the method converges for merely $L^{\infty}$ uniformly elliptic tensor $\mathbf{A}(\cdot)$, it is not designed for a smart handling of a piecewise continuous $\mathbf{A}(\cdot)^{5}$. Therefore, we skip Test 5 that involves piecewise constant $\mathbf{A}(\cdot)$. We refer to Coudière, Pierre, Rousseau and Turpault [12] and to Hermeline [17] for 3D CeVe-DDFV constructions efficiently taking into account discontinuities of $\mathbf{A}(\cdot)$.

Choice of the cell and face points. We pick for $x_{K}$, the isobarycenter of the cell $K$, and for $x_{K L L}$, the isobarycenter of the face $K \mid L$.

\footnotetext{
${ }^{4}$ The restriction on the number $l$ of face vertices is only needed for justifying a discrete Poincaré inequality; yet this property is immaterial, e.g., for the associated evolution problem. In practice, in the below tests values $l=3,4,6$ were used, and no particular problem for $l=6$ is reported.

${ }^{5}$ In 2D, a scheme called m-DDFV, specifically designed to handle discontinuous diffusion tensors, was designed by Boyer and Hubert in [6]. There is a clear difference in convergence orders for the basic DDFV version [5] and the m-DDFV version [6] (see the 2D benchmark paper [7]).
} 
Measure of errors and convergence orders. To put the discrete and the exact solutions "at the same level", we use the projection $\mathbb{P}^{\mathfrak{T}} u_{e}$ of the exact solution and the associated discrete gradient reconstruction $\vec{\nabla}^{\mathfrak{I}} \mathbb{P}^{\overline{\mathfrak{s}}} u_{e}$, where $\mathbb{P}^{\overline{\mathfrak{x}}} \cdot=\left(\mathbb{P}^{\mathfrak{T}} \cdot ; \mathbb{P}^{\partial \mathfrak{T}} \cdot\right)$. The

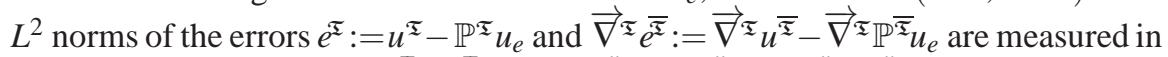
terms of the scalar products $\llbracket \cdot, \cdot \rrbracket$ on $\mathbb{R}^{\mathfrak{T}},\left\{\left\{\mathbf{A}^{\mathfrak{T}} \cdot, \cdot\right\}\right.$ and $\left.\{\cdot, \cdot\}\right\}$ on $\left(\mathbb{R}^{3}\right)^{\mathfrak{D}}$ : the relative error indicators erl2 and ener, ergrad we use are defined, respectively, as

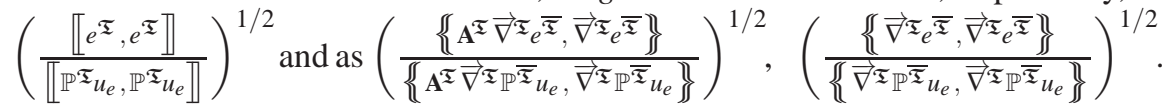

- Test 1 Mild anisotropy, $u_{e}(x, y, z)=1+\sin (\pi x) \sin \left(\pi\left(y+\frac{1}{2}\right)\right) \sin \left(\pi\left(z+\frac{1}{3}\right)\right)$ $\min =0, \max =2$, Tetrahedral meshes

\begin{tabular}{|c|ccccccc|}
$\mathrm{i}$ & $\mathrm{nu}$ & $\mathrm{nmat}$ & umin & uemin & umax & uemax & normg \\
\hline 1 & 2187 & 21287 & $0.706 \mathrm{E}-02$ & $0.706 \mathrm{E}-02$ & 1.992 & 1.992 & $0.178 \mathrm{E}+01$ \\
\hline 2 & 4301 & 44813 & $0.706 \mathrm{E}-02$ & $0.706 \mathrm{E}-02$ & 1.997 & 1.996 & $0.179 \mathrm{E}+01$ \\
\hline 3 & 8584 & 94088 & $0.278 \mathrm{E}-02$ & $0.278 \mathrm{E}-02$ & 1.993 & 1.993 & $0.179 \mathrm{E}+01$ \\
\hline 4 & 17102 & 195074 & $0.792 \mathrm{E}-03$ & $0.792 \mathrm{E}-03$ & 1.997 & 1.997 & $0.179 \mathrm{E}+01$ \\
\hline 5 & 34343 & 405077 & $0.140 \mathrm{E}-02$ & $0.140 \mathrm{E}-02$ & 1.999 & 1.999 & $0.180 \mathrm{E}+01$ \\
\hline 6 & 69160 & 838856 & $0.140 \mathrm{E}-02$ & $0.140 \mathrm{E}-02$ & 1.999 & 1.999 & $0.180 \mathrm{E}+01$ \\
\hline
\end{tabular}

\begin{tabular}{|c|ccccccc|} 
i & nu & erl2 & ratiol2 & ergrad & ratiograd & ener & ratioener \\
\hline 1 & 2187 & $0.539 \mathrm{E}-02$ & - & $0.654 \mathrm{E}-01$ & - & $0.649 \mathrm{E}-01$ & - \\
\hline 2 & 4301 & $0.331 \mathrm{E}-02$ & 2.165 & $0.488 \mathrm{E}-01$ & 1.297 & $0.491 \mathrm{E}-01$ & 1.239 \\
\hline 3 & 8584 & $0.206 \mathrm{E}-02$ & 2.069 & $0.381 \mathrm{E}-01$ & 1.077 & $0.383 \mathrm{E}-01$ & 1.079 \\
\hline 4 & 17102 & $0.135 \mathrm{E}-02$ & 1.841 & $0.301 \mathrm{E}-01$ & 1.018 & $0.302 \mathrm{E}-01$ & 1.026 \\
\hline 5 & 34343 & $0.846 \mathrm{E}-03$ & 1.998 & $0.240 \mathrm{E}-01$ & 0.973 & $0.242 \mathrm{E}-01$ & 0.955 \\
\hline 6 & 69160 & $0.539 \mathrm{E}-03$ & 1.934 & $0.190 \mathrm{E}-01$ & 1.012 & $0.191 \mathrm{E}-01$ & 1.008 \\
\hline
\end{tabular}

- Test 1 Mild anisotropy, $u_{e}(x, y, z)=1+\sin (\pi x) \sin \left(\pi\left(y+\frac{1}{2}\right)\right) \sin \left(\pi\left(z+\frac{1}{3}\right)\right)$ $\min =0, \max =2$, Voronoi meshes

\begin{tabular}{|c|cccccc|c|}
$\mathrm{i}$ & $\mathrm{nu}$ & nmat & umin & uemin & umax uemax & normg \\
\hline 1 & 87 & 1433 & $0.667 \mathrm{E}-01$ & $0.667 \mathrm{E}-01$ & 1.904 & 1.904 & $0.159 \mathrm{E}+01$ \\
\hline 2 & 235 & 4393 & $0.432 \mathrm{E}-02$ & $0.432 \mathrm{E}-02$ & 1.997 & 1.997 & $0.172 \mathrm{E}+01$ \\
\hline 3 & 527 & 10777 & $0.280 \mathrm{E}-01$ & $0.280 \mathrm{E}-01$ & 1.990 & 1.990 & $0.176 \mathrm{E}+01$ \\
\hline 4 & 1013 & 21793 & $0.108 \mathrm{E}-02$ & $0.108 \mathrm{E}-02$ & 2.003 & 1.995 & $0.177 \mathrm{E}+01$ \\
\hline 5 & 1776 & 40998 & $0.113 \mathrm{E}-01$ & $0.113 \mathrm{E}-01$ & 2.000 & 1.996 & $0.178 \mathrm{E}+01$ \\
\hline
\end{tabular}

\begin{tabular}{|c|ccccccc|} 
ii & nu & erl2 & ratiol2 & ergrad & ratiograd & ener & ratioener \\
\hline 1 & 87 & $0.484 \mathrm{E}-01$ & - & $0.204 \mathrm{E}+00$ & - & $0.374 \mathrm{E}+00$ & - \\
\hline 2 & 235 & $0.388 \mathrm{E}-01$ & 0.666 & $0.173 \mathrm{E}+00$ & 0.496 & $0.277 \mathrm{E}+01$ & -6.049 \\
\hline 3 & 527 & $0.231 \mathrm{E}-01$ & 1.925 & $0.118 \mathrm{E}+00$ & 1.402 & $0.838 \mathrm{E}+00$ & 4.445 \\
\hline 4 & 1013 & $0.167 \mathrm{E}-01$ & 1.484 & $0.940 \mathrm{E}-01$ & 1.060 & $0.299 \mathrm{E}+01$ & -5.843 \\
\hline 5 & 1776 & $0.117 \mathrm{E}-01$ & 1.937 & $0.818 \mathrm{E}-01$ & 0.742 & $0.291 \mathrm{E}+01$ & 0.147 \\
\hline
\end{tabular}


- Test 1 Mild anisotropy, $u_{e}(x, y, z)=1+\sin (\pi x) \sin \left(\pi\left(y+\frac{1}{2}\right)\right) \sin \left(\pi\left(z+\frac{1}{3}\right)\right)$ $\min =0, \max =2$, Kershaw meshes

\begin{tabular}{|c|c|c|c|c|}
\hline $\mathrm{nu}$ & nmat & uemin umax & uemax & normg \\
\hline 855 & 13819 & $2.28 \mathrm{E}-02$ 2.28E-02 1.989 & 1.989 & 1.730 \\
\hline 7471 & 138691 & $2.52 \mathrm{E}-03$ 2.52E-03 1.994 & 1.994 & 1.778 \\
\hline 62559 & 1237459 & 1.99E-03 1.99E-03 1.999 & 1.999 & 1.794 \\
\hline \begin{tabular}{l|l}
4 & 512191
\end{tabular} & 10443763 & $3.82 \mathrm{E}-04$ 3.82E-04 2.000 & 2.000 & 1.797 \\
\hline
\end{tabular}

\begin{tabular}{|c|ccccccc|}
$\mathrm{i}$ & $\mathrm{nu}$ & erl2 & ratiol2 & ergrad & ratiograd & ener & ratioener \\
\hline 1 & 855 & $0.501 \mathrm{E}-01$ & - & $0.484 \mathrm{E}+00$ & - & $0.558 \mathrm{E}+00$ & - \\
\hline 2 & 7471 & $0.156 \mathrm{E}-01$ & 1.611 & $0.209 \mathrm{E}+00$ & 1.160 & $0.159 \mathrm{E}+00$ & 1.735 \\
\hline 3 & 62559 & $0.392 \mathrm{E}-02$ & 1.954 & $0.677 \mathrm{E}-01$ & 1.594 & $0.395 \mathrm{E}-01$ & 1.970 \\
\hline 4 & 512191 & $0.101 \mathrm{E}-02$ & 1.936 & $0.223 \mathrm{E}-01$ & 1.585 & $0.109 \mathrm{E}-01$ & 1.835 \\
\hline
\end{tabular}

- Test 1 Mild anisotropy, $u_{e}(x, y, z)=1+\sin (\pi x) \sin \left(\pi\left(y+\frac{1}{2}\right)\right) \sin \left(\pi\left(z+\frac{1}{3}\right)\right)$ $\min =0, \max =2$, Checkerboard meshes

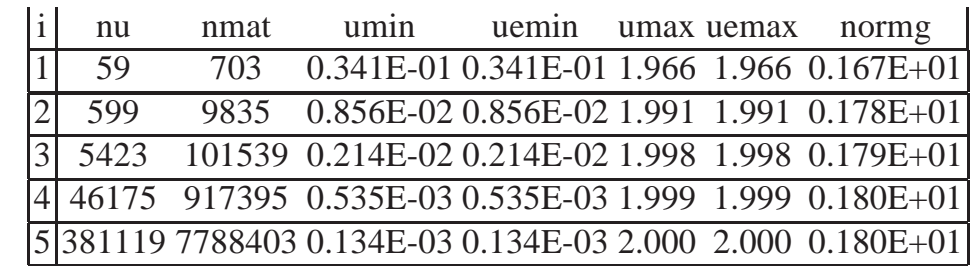

\begin{tabular}{|c|ccccccc|} 
i & nu & erl2 & ratiol2 & ergrad & ratiograd & ener & ratioener \\
\hline 1 & 59 & $0.396 \mathrm{E}-01$ & - & $0.136 \mathrm{E}+00$ & - & $0.116 \mathrm{E}+00$ & - \\
\hline 2 & 599 & $0.149 \mathrm{E}-01$ & 1.266 & $0.928 \mathrm{E}-01$ & 0.499 & $0.818 \mathrm{E}-01$ & 0.449 \\
\hline 3 & 5423 & $0.400 \mathrm{E}-02$ & 1.792 & $0.497 \mathrm{E}-01$ & 0.849 & $0.448 \mathrm{E}-01$ & 0.820 \\
\hline 4 & 46175 & $0.103 \mathrm{E}-02$ & 1.905 & $0.256 \mathrm{E}-01$ & 0.931 & $0.232 \mathrm{E}-01$ & 0.920 \\
\hline 5 & 381119 & $0.259 \mathrm{E}-03$ & 1.954 & $0.130 \mathrm{E}-01$ & 0.965 & $0.118 \mathrm{E}-01$ & 0.961 \\
\hline
\end{tabular}

- Test 2 Heterogeneous anisotropy, $\min =-0.862, \max =1.0487$

$u_{e}(x, y, z)=x^{3} y^{2} z+x \sin (2 \pi x z) \sin (2 \pi x y) \sin (2 \pi z)$, Prism meshes

\begin{tabular}{|c|cccccc|c|}
$\mathrm{i}$ & $\mathrm{nu}$ & $\mathrm{nmat}$ & umin & uemin & umax & uemax & normg \\
\hline 1 & 3010 & 64158 & $-.856 \mathrm{E}+00$ & $-.856 \mathrm{E}+00$ & 1.044 & 1.044 & $0.170 \mathrm{E}+01$ \\
\hline 2 & 24020 & 555528 & $-.859 \mathrm{E}+00$ & $-.859 \mathrm{E}+00$ & 1.047 & 1.047 & $0.171 \mathrm{E}+01$ \\
\hline 3 & 81030 & 1924098 & $-.861 \mathrm{E}+00$ & $-.861 \mathrm{E}+00$ & 1.049 & 1.049 & $0.171 \mathrm{E}+01$ \\
\hline 4 & 192040 & 4619868 & $-.862 \mathrm{E}+00$ & $-.862 \mathrm{E}+00$ & 1.049 & 1.049 & $0.171 \mathrm{E}+01$ \\
\hline
\end{tabular}

\begin{tabular}{|c|c|c|c|c|c|c|c|}
\hline & $\mathrm{nu}$ & erl2 & ratiol2 & ergrad & ratiograd & ener & ratioener \\
\hline & 3010 & $0.467 \mathrm{E}-01$ & - & $0.711 \mathrm{E}-01$ & - & $0.785 \mathrm{E}-01$ & \\
\hline 2 & 24020 & $0.123 \mathrm{E}-01$ & 1.931 & $0.224 \mathrm{E}-01$ & 1.667 & $0.328 \mathrm{E}-01$ & 1.262 \\
\hline 3 & 81030 & $0.554 \mathrm{E}-02$ & 1.960 & $0.116 \mathrm{E}-01$ & 1.634 & $0.190 \mathrm{E}-01$ & 1.348 \\
\hline & 192040 & $0.314 \mathrm{E}-02$ & 1.973 & $0.728 \mathrm{E}-02$ & 1.607 & $0.127 \mathrm{E}-01$ & 1.389 \\
\hline
\end{tabular}


- Test 3 Flow with strong anisotropy on random meshes, $\min =0, \max =1$, $u_{e}(x, y, z)=\sin (\pi x) \sin (\pi y) \sin (\pi z)$, Random meshes

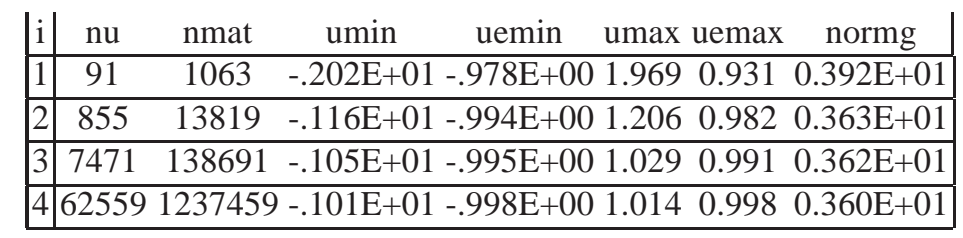

\begin{tabular}{|c|ccccccc|}
$\mathrm{i}$ & $\mathrm{nu}$ & erl2 & ratiol2 & ergrad & ratiograd & ener & ratioener \\
\hline 1 & 91 & $0.713 \mathrm{E}+00$ & - & $0.716 \mathrm{E}+00$ & - & $0.439 \mathrm{E}+00$ & - \\
\hline 2 & 855 & $0.152 \mathrm{E}+00$ & 2.068 & $0.199 \mathrm{E}+00$ & 1.712 & $0.130 \mathrm{E}+00$ & 1.633 \\
\hline 3 & 7471 & $0.384 \mathrm{E}-01$ & 1.906 & $0.854 \mathrm{E}-01$ & 1.174 & $0.417 \mathrm{E}-01$ & 1.568 \\
\hline 4 & 62559 & $0.119 \mathrm{E}-01$ & 1.656 & $0.542 \mathrm{E}-01$ & 0.640 & $0.183 \mathrm{E}-01$ & 1.165 \\
\hline
\end{tabular}

- Test 4 Flow around a well, $\min =0, \max =5.415$, Well meshes

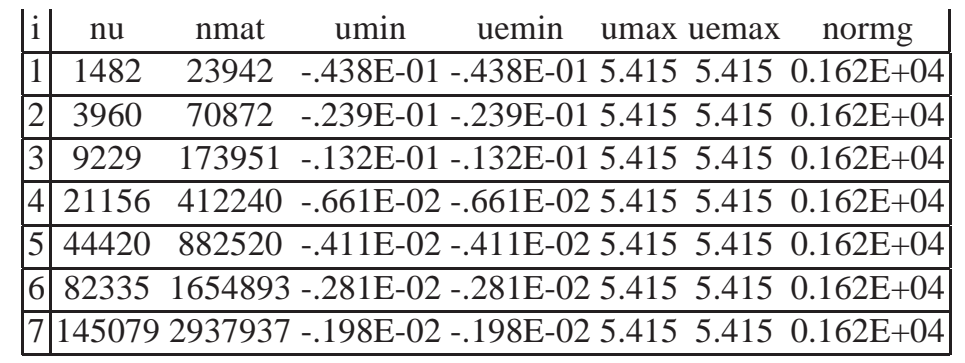

\begin{tabular}{|c|ccccccc|}
$\mathrm{i}$ & $\mathrm{nu}$ & erl2 & ratiol2 & ergrad & ratiograd & ener & ratioener \\
\hline 1 & 1482 & $0.564 \mathrm{E}-02$ & - & $0.473 \mathrm{E}-01$ & - & $0.817 \mathrm{E}-01$ & - \\
\hline 2 & 3960 & $0.218 \mathrm{E}-02$ & 2.897 & $0.205 \mathrm{E}-01$ & 2.556 & $0.487 \mathrm{E}-01$ & 1.578 \\
\hline 3 & 9229 & $0.964 \mathrm{E}-03$ & 2.898 & $0.108 \mathrm{E}-01$ & 2.255 & $0.296 \mathrm{E}-01$ & 1.770 \\
\hline 4 & 21156 & $0.645 \mathrm{E}-03$ & 1.454 & $0.748 \mathrm{E}-02$ & 1.344 & $0.205 \mathrm{E}-01$ & 1.320 \\
\hline 5 & 44420 & $0.427 \mathrm{E}-03$ & 1.664 & $0.546 \mathrm{E}-02$ & 1.274 & $0.144 \mathrm{E}-01$ & 1.443 \\
\hline 6 & 82335 & $0.291 \mathrm{E}-03$ & 1.864 & $0.396 \mathrm{E}-02$ & 1.560 & $0.108 \mathrm{E}-01$ & 1.391 \\
\hline 7 & 145079 & $0.205 \mathrm{E}-03$ & 1.848 & $0.337 \mathrm{E}-02$ & 0.858 & $0.794 \mathrm{E}-02$ & 1.624 \\
\hline
\end{tabular}

\section{Comments}

Let us summarize the observations; footnotes provide comments of theoretical order.

Choice of the solvers. The following results have been performed either with the direct solvers given by the UMFPACK library, or with the BiCGStab algorithm with ILU(0) preconditionning delivered in the HSL library. A comparison between ISTLCG with ILU(0) preconditionning and PETSC-CG with ILU(2) preconditionning shows that, whenever ISTL-CG/ILU(0) algorithm converges, much less CPU time and much less memory is used than for the PETSC-CG/ILU(2) algorithm. 
Convergence orders observed ${ }^{6}$. Even if the orders present serious oscillations for some cases (e.g., in Test 3 and in Test 1 on Voronoï meshes), orders slightly below $h^{2}$ (superconvergence) for the solution in the $L^{2}$ norm are observed quite systematically. One exception is Test 4 , where an order intermediate between $h^{3 / 2}$ and $h^{2}$ seems to appear; this may be related to the presence of a singularity in the well center.

Regarding the gradient norm, convergence orders close to $h$ are seen in Test 1 on tetrahedral, Voronoï, checkerboard meshes. On Kershaw meshes in Test 1 and prism meshes of Test 2 , more structured though distorted, an $h^{3 / 2}$ convergence order can be observed. For random meshes of Test 3 , orders degrade quickly but the numerical evidence (four meshes only) seems insufficient. The well meshes of Test 4 appear as rather structured but having a singularity; the effect of singularity grows as the mesh becomes finer, and the convergence order falls from $h^{2}$ to $h^{3 / 2}$ and then to $h$. Yet from Tests 3 and 4 with stronger anisotropy of $\mathbf{A}(\cdot)$, it becomes clear that more adequate norm for measuring gradient convergence is the energy norm. In Test 4 we observe an accurate $h^{3 / 2}$ convergence and in Test 3 , an order $h^{3 / 2}$ can be conjectured.

Violation (and fulfillment) of the maximum principle ${ }^{7}$. We observe that violation of discrete maximum principle does not occur systematically (or if it occurs, it is of imperceptible magnitude, even on coarse meshes). No overshoot/undershoot is reported on Kershaw, checkerboard and prism meshes for Test 1, nor on the well meshes of Test 4; a very slight overshoot can be seen in Test 1 on tetrahedral meshes. On the contrary, random meshes of Test 3 , and also the finest ones among the Voronoï meshes of Test 1, exhibit a perceptible violation of the maximum principle which is nonetheless reduced as the mesh size diminishes ${ }^{8}$. Difficulties on these two kinds of meshes can be explained by their poor shape regularity (e.g., fine Voronoï meshes in Test 1 present a dramatic contrast of size between neighbor cells).

Influence of the mesh type and quality on convergence orders ${ }^{9}$. Among the different mesh properties that could influence the numerical behavior, restrictions on $l$ appear as immaterial (the best convergence orders are achieved for prism meshes of Test 2 having up to $l=6$ face vertices). While conformity is not needed for the method, non-conformal meshes bring more distorted cells and diamonds. We have seen that bad shape conditioning may induce violation of the maximum principle. In Test 1, presence of neighbor cells with considerable contrast in size (for Voronoï meshes and for non-conformal checkerboard meshes) degrades convergence orders for the gradient, in contrast to rather gradually distorted Kershaw and prism meshes.

${ }^{6}$ For regular enough $\mathbf{A}(\cdot)$ and $u_{e}$, order $h$ can be proved for both solution and its gradient in $L^{2}$.

${ }^{7}$ In principle, DDFV methods are not designed in order to respect the discrete maximum principle; and the convergence analysis exploits rather the variational structure, well preserved by the method (this is one of the benefits from the discrete duality of Prop. 1). Let us point out that for isotropic problems on primal meshes satisfying the orthogonality condition (e.g., Delaunay tetrahedral meshes with the choice of circumcenters for the cell centers $x_{K}$ - note that $x_{K}$ may fall out of $K$ ), the discrete maximum principle is easily shown for the CeVe-DDFV scheme under study ([4]).

${ }^{8}$ In theory, one can prove convergence in $L^{q}$ for $q<6$; nothing guarantees convergence in $L^{\infty}$.

${ }^{9}$ Recall that conformity of meshes is not required by the method; and the construction allows for unrestricted number $l$ of face vertices. Yet it is a well-known difficulty for the analysis of the scheme that the discrete Poincaré inequality cannot be proved for $l>4$, see $[17,2]$. 


\section{References}

1. B. Andreianov, M. Bendahmane, F. Hubert and S. Krell. On 3D DDFV discretization of gradient and divergence operators. I. Meshing, operators and discrete duality. Preprint HAL (2011), http://hal.archives-ouvertes.fr/hal-00355212.

2. B. Andreianov, M. Bendahmane and F. Hubert. On 3D DDFV discretization of gradient and divergence operators. II. Discrete functional analysis tools and applications to degenerate parabolic problems. Preprint HAL (2011), http://hal.archives-ouvertes.fr/hal-00567342.

3. B. Andreianov, M. Bendahmane, and K. Karlsen. A gradient reconstruction formula for finite-volume schemes and discrete duality. In R. Eymard and J.-M. Hérard, editors, Finite Volume For Complex Applications, Problems And Perspectives. 5th International Conference, (2008),161-168. London (UK) Wiley.

4. B. Andreianov, M. Bendahmane, and K.H. Karlsen. Discrete duality finite volume schemes for doubly nonlinear degenerate hyperbolic-parabolic equations. J. Hyperbolic Diff. Equ. (2010), 7(1):1-67.

5. B. Andreianov, F. Boyer, and F. Hubert. Discrete duality finite volume schemes for Leray-Lions type elliptic problems on general 2D-meshes. Numer. Methods PDE, (2007), 23(1):145-195.

6. F. Boyer and F. Hubert. Finite volume method for 2D linear and nonlinear elliptic problems with discontinuities. SIAM J. Num. Anal., (2008), 46(6):3032-3070.

7. F. Boyer and F. Hubert. Benchmark on anisotropic problems, the ddfv discrete duality finite volumes and m-ddfv schemes. In R. Eymard and J.-M. Hérard, editors, Finite Volume For Complex Applications, Problems And Perspectives. 5th International Conference, (2008), 735-750. London (UK) Wiley.

8. Y. Coudière, J.-P. Vila, and P. Villedieu. Convergence rate of a finite volume scheme for a two dimensional convection-diffusion problem. M2AN Math. Modelling Num. Anal., (1999), 33(3):493-516.

9. Y. Coudière and C. Pierre. Benchmark 3D: CeVe-DDFV, a discrete duality scheme with cell/vertex unknowns. Proc. FVCA6 (Prague), (2011); available as preprint HAL (2011), http://hal.archives-ouvertes.fr/hal-00561980

10. Y. Coudière and F. Hubert. A 3D discrete duality finite volume method for nonlinear elliptic equation. preprint (2009), http://hal.archives-ouvertes.fr/hal-00456837

11. Y. Coudière, F. Hubert and G. Manzini. Benchmark 3D: CeVeFE-DDFV, a discrete duality scheme with cell/vertex/face+edge unknowns. Proc. FVCA6 (Prague), (2011).

12. Y. Coudière, C. Pierre, O. Rousseau, and R. Turpault. A 2d/3d discrete duality finite volume scheme. Application to ecg simulation. Int. Journal on Finite Volumes, (2009), 6(1).

13. K. Domelevo and P. Omnès. A finite volume method for the Laplace equation on almost arbitrary two-dimensional grids. M2AN Math. Model. Numer. Anal., (2005), 39(6):12031249.

14. R. Herbin and F. Hubert. Benchmark on discretization schemes for anisotropic diffusion problems on general grids. In R. Eymard and J.-M. Hérard, editors, Finite Volume For Complex Applications, Problems And Perspectives. 5th International Conference, (2008), 659-692. London (UK) Wiley.

15. F. Hermeline. A finite volume method for the approximation of diffusion operators on distorted meshes. J. Comput. Phys., (2000), 160(2):481-499.

16. F. Hermeline. Approximation of 2-d and 3-d diffusion operators with variable full tensor coefficients on arbitrary meshes. Comput. Methods Appl. Mech. Engrg., (2007), 196(2124):2497-2526.

17. F. Hermeline. A finite volume method for approximating $3 \mathrm{~d}$ diffusion operators on general meshes. Journal of computational Physics, (2009), 228(16):5763-5786.

18. S. Krell. Schémas Volumes Finis en mécanique des fluides complexes. Ph.D. Thesis, Univ. de Provence, Marseilles, (2010), http://tel.archives-ouvertes.fr/tel-00524509.

19. S. Krell. Stabilized DDFV schemes for Stokes problem with variable viscosity on general 2D meshes. Num. Meth. PDEs, (2010), available on-line: http://dx.doi.org/10.1002/num.20603. 